\title{
Pandemia do coronavírus e impactos na saúde mental: uma revisão integrativa da literatura
}

\author{
Coronavirus pandemic and impacts on mental \\ health: an integrative literature review
}

\section{Angélica Vanessa de Andrade Araújo Lira1 ${ }^{10}$ Nilza Alessandra Pereira² (1) Luís Augusto Irineu Aguiar Ramos ${ }^{3}$ (1) Clésia Oliveira Pachú 4 (1)}

\footnotetext{
1Autora para correspondência. Universidade Estadual da Paraíba (Campina Grande). Paraíba, Brasil. angelicavanessa14@gmail.com

2,3Universidade Estadual da Paraíba (Campina Grande). Paraíba, Brasil. alessandracardosonp@gmail.com, luismedufcg@gmail.com ${ }^{4}$ Universidade Federal de Campina Grande, Universidade Estadual da Paraíba (Campina Grande). Paraíba, Brasil. clesiapachu@hotmail.com
}

RESUMO | Em cenários de pandemia, como a COVID-19, a ruptura da cadeia de infecção é fundamental para a proteção da saúde da população. Este processo pode ser atingido mediante a adoção de medidas de restrição do contato humano, como isolamento, quarentena e distanciamento social. Embora fundamentadas em achados científicos e necessárias para conter o avanço da COVID-19, as medidas de isolamento social pode gerar impactos à saúde mental da população. Nesse sentido, a presente pesquisa visa avaliar a produção científica a respeito dos impactos da quarentena decorrente da COVID-19 na saúde mental. Trata-se de uma revisão integrativa da literatura científica, realizada por meio da exploração nas bases de dados PubMed e Biblioteca Virtual em Saúde (BVS), no período de março de 2020. Nessas bases de dados, foram utilizadas as palavraschave: COVID-19 and psychological consequences, COVID-19 and psychological impact of quarantine, obteve-se 10 artigos, dos quais 4 artigos, foram incluídos na análise após a exclusão de artigos duplicados e revisões de literatura, sendo 3 da PubMed e 1 da BVS. Os resultados sugerem alta frequência de relatos de reações e sintomas possivelmente associados a transtornos mentais, tais como queixas de ansiedade, depressão, insônia, estresse, pânico, solidão, angústia, obsessão e compulsão entre outros. Ficou evidente que grupos de risco como idosos e pessoas com doenças crônicas apresentaram maior probabilidade para o desenvolvimento de transtornos mentais. Ademais, análises dos impactos do coronavírus na dimensão psicológica podem corroborar para construção de intervenções pautadas na promoção, prevenção e tratamento em saúde mental.

PALAVRAS-CHAVE: Coronavírus. Transtornos Mentais. Saúde Mental. Saúde Pública.
ABSTRACT I In pandemic scenarios, such as COVID-19, the disruption of the infection chain is fundamental for the protection of the population's health. This process can be achieved through the adoption of measures to restrict human contacts, such as isolation, quarantine, and social distance. Although based on scientific findings and necessary to contain the advancement of COVID-19, social isolation measures can have an impact on the population's mental health. In this sense, this research aims to evaluate scientific production regarding the impacts of the quarantine resulting from COVID-19 on mental health. It is an integrative review of the scientific literature, carried out through the exploration in the PubMed and Virtual Health Library (BVS) databases, in March 2020. In these databases, the keywords were used: COVID -19 and psychological consequences, COVID-19 and psychological impact of quarantine, 10 articles were obtained, of which 4 articles were included in the analysis after excluding duplicate articles and literature reviews, 3 from PubMed and 1 from the BVS. The results suggest a high frequency of reports of reactions and symptoms possibly associated with mental disorders, such as complaints of anxiety, depression, insomnia, stress, panic, loneliness, anguish, obsession, and compulsion, among others. It was evident that risk groups such as the elderly and people with chronic diseases were more likely to develop mental disorders. In addition, analyzes of the impact of coronavirus on the psychological dimension can support the construction of interventions based on promotion, prevention, and treatment in mental health.

KEYWORDS: Coronavirus. Mental Disorders. Mental Health. Public Health. 


\section{Introdução}

A COVID-19, enquanto pandemia, promoveu impactos na saúde mental dos indivíduos, conduzindo-os a adotarem medidas de separação física, como isolamento, quarentena e distanciamento social. Embora semelhantes, essas importantes estratégias de intervenções no âmbito da saúde pública não são sinônimas. Em relação ao isolamento, vê-se que é a separação da população doente daquelas que não estão infectadas, tendo como finalidade reduzir o risco de transmissão (Wilder-Smith \& Freedman, 2020). Quanto à quarentena, por sua vez, é a separação e a restrição da movimentação do indivíduo a espaços de possíveis contágios. O distanciamento social relaciona-se na conscientização pessoal da coletividade, a fim de reduzir o contato e aumentar a distância física entre a população, para a diminuição da velocidade do contágio (Fiocruz, 2020). Considerando que essas medidas se convergem ao mesmo propósito de conter o avanço da COVID-19 no cenário social, no presente estudo, todas essas expressões serão usadas com os significados similares à logística de quarentena doméstica.

A Doença do Vírus Corona 2019 (COVID-19) é uma doença altamente infecciosa com um longo período de incubação causado pelo Coronavírus 2 da Síndrome Aguda Grave (Sars-Cov-2) (Huang et al., 2020). O primeiro caso da patologia da COVID-19 foi registrado em Wuhan, na China, no dia 31 de dezembro de 2019, e se espalhou rapidamente, nacional e internacionalmente (World Health Organization [WHO], 2020; WHO, 2020), ocasionou crescente atenção tanto das autoridades públicas quanto da sociedade civil. No Brasil, o primeiro caso notificado de COVID-19 ocorreu em São Paulo, em fevereiro de 2020, envolvendo um homem de 61 anos com histórico de viagem para a Itália; e, logo após, se alastrou para os demais estados brasileiros (Ministério da Saúde [MS], 2020).

Por assumir conotação de ordem mundial, o COVID-19 foi considerado pela Organização Mundial da Saúde (OMS), em 31 de janeiro de 2020, como emergência de saúde pública de preocupação internacional (PHEIC) (WHO, 2020), e, em 11 de março do mesmo ano, foi declarado oficialmente como pandemia (WHO, 2020). Neste sentido, trata-se de um fenômeno patológico que alterou profundamente a dinâmica de organização da sociedade, fomentando sérios danos, não apenas ao sistema de saúde, como também, implicações de ordem econômica, social e política.

Clinicamente, vê-se que os coronavírus causam deficiências respiratórias. Comumente, ocorre com mais frequência à infecção pelos coronavírus denominados de alpha coronavírus 229E e NL63 e beta coronavírus OC43, HKU1 (MS, 2020). O quadro clínico pode ser desde um resfriado comum até doenças mais graves como a Síndrome Respiratória do Oriente Médio (MERS) e a Síndrome Respiratória Aguda Grave (SARS). Observa-se que, grande parte dos casos de SARS progride para pneumonia (Huang et al., 2020). Em geral, o tempo de incubação ocorre em média de 5 a 6 dias, podendo atingir até 14 dias (WHO, 2020) onde a transmissão em humanos ocorre por meio do contato com secreções contaminadas. Neste contexto, o diagnóstico pode ser realizado mediante amostras de sangue, fezes ou secreções nasais, utilizando-se de testes sorológicos, Reação em Cadeia da Polimerase (PCR) ou isolamento viral (WHO, 2020).

Assim, em se tratando de uma patologia de fácil transmissão, com incidência alta de letalidade em grupos de risco como idosos e pessoas com doenças crônicas (WHO, 2020), autoridades públicas adotaram uma série de medidas restritivas de quarentena, no sentido de evitar e minimizar o contágio do vírus entre a coletividade. Dessa forma, ocorreram restrições de circulação urbana, fechamentos de locais de entretenimento e lazer, pânico coletivo gerado pela divulgação diária de notícias em meios midiáticos acerca do coronavírus, perdas de entes queridos, impossibilidade de visitar amigos e familiares que corroboram no crescimento de patologias mentais na população como transtorno do pânico, ansiedade e depressão (Qiu et al., 2020). Pôde-se observar que a instituição do isolamento social alterou de forma abrupta a rotina diária de grande parte da população.

Essas medidas de distanciamento social, além de impossibilitar o contato face a face entre as pessoas, delinearam condições limitantes para o exercício pleno da liberdade humana, impondo mudanças na rotina habitual das pessoas e a exigência de adoção de novos hábitos sociais como o uso da máscara em locais públicos, supressão de atos de saudações (apertos de mão e beijos no rosto), entre outros. Esse cenário de limitação da expressão das vivências habituais humanas desencadeou nas pessoas dificuldades de adaptação a essa nova realidade social (Li et al., 2020). 
Em decorrência disso, a população está imbuída em um contexto de instabilidade econômica, política e social. Nesse ínterim, as pessoas convivem diariamente com a insegurança e o medo de contrair o vírus e, por vezes, com a incerteza e a falta de informações concisas para o retorno à vida normal, delineando um cenário favorável para a configuração de quadros de sofrimento existencial e social (Qiu et al., 2020). Dessa forma, a vigência de medidas de isolamento no cenário social pode desencadear o desenvolvimento de sensações de distanciamento emocional e restrição da liberdade por quem as experiencia.

Nesse sentido, as medidas de isolamento social são frequentemente associadas as experiências desagradáveis caraterizadas por mudanças bruscas e, muitas vezes, indesejadas do cotidiano das pessoas, implicando em uma série de variáveis restritivas que ocasionam rupturas com a produtividade da lógica capitalista e a vida frenética e imediatista da sociedade. Trata-se de um contexto responsável por alterações profundas em diferentes esferas da vida humana como trabalho, família, educação, lazer, interação comunitária entre outros. Esse cenário pandêmico mostra a vulnerabilidade da existência humana e reitera a incapacidade do homem de lidar com o inesperado e, sobretudo a dificuldade de se desprender da rotina materialista e produtiva da organização societal (Qiu et al., 2020).

Durante a execução das medidas de isolamento social, as pessoas são atravessadas por uma série de estressores que dificultam o processo adaptativo ao novo cenário social como duração prolongada dessas medidas, o medo iminente de ser infectado e infectar seus entes queridos, a monotonia da rotina diária embalada pelo tédio e frustração, dificuldades para a obtenção ou fragilidade de suporte socioafetivo, informações vagas e imprecisas sobre o coronavírus e falta de suprimentos adequados para lidar com esse cenário de pandemia (Fiocruz, 2020). Esses agentes estressores podem aumentar a incidência de quadros de sofrimento psicológico na população.

Nessa perspectiva, o fenômeno COVID-19 fomenta implicações negativas à condição psicológica da população. Isso concorre na configuração de processos intrincados de subjetivação e menor capacidade responsiva, permeada pelo clima de pressão psicológica. Assim, pacientes, profissionais de saúde e público em geral estão vulneráveis ao poder desagregador de sentimentos negativos que acompanham a luta pela preservação física em relação ao coronavírus com prejuízos nas esferas da vida - laboral, educacional, afetiva, familiar e social. A ausência de terapia efetiva e a incerteza da cura permeia o imaginário social, reverberando a existência de um pré-adoecimento, isto é, a doença provoca sofrimento antes mesmo de instalar-se no organismo (Qiu et al., 2020).

É inegável, o coronavírus vem se mostrando como grave problema de saúde pública, fomentando implicações de ordem clínica, psicológica, social e econômica na vida de pacientes, profissionais de saúde e população em geral (WHO, 2020; WHO, 2020; WHO, 2020). Considerando as implicações psicológicas dessa patologia, evidencia-se a importância de discutir e explicar a dinâmica processual dos efeitos e consequências geradas pelo vírus, bem como os impactos das mudanças sociais influenciada por essa patologia, identificando os elementos desse fenômeno que corroboram para o desencadeamento e potencialização de transtornos mentais.

Dada a relevância da pandemia COVID-19 no cenário mundial e a ausência de produções científicas no cenário nacional acerca da referida temática, justifica-se a necessidade do presente estudo. Partindo dessa perspectiva, o estudo pode servir como base para ampliação de novas produções científicas nessa área, bem como um instrumento importante para subsidiar a criação de estratégias interventivas acionáveis no campo de saúde voltadas à promoção e tratamento em saúde mental. Mediante o exposto, a presente pesquisa visa avaliar a produção científica a respeito dos impactos da quarentena decorrente da COVID-19 na saúde mental.

\section{Métodos}

A pesquisa consiste em uma revisão integrativa de literatura científica, sendo subdividida em cinco fases: elaboração da pergunta norteadora da busca ou amostragem na literatura, coleta de dados, análise crítica dos estudos incluídos, discussão dos resultados e apresentação da revisão integrativa (Souza \& Carvalho, 2010). Para tanto, realizou-se o levantamento bibliográfico, com abordagem quantitativa e qualitativa, em relação à pergunta norteadora da pesquisa: Qual o impacto da pandemia da COVID-19 para a saúde mental? A busca na literatura foi realizada na base de dados PubMed e na plataforma de dados Biblioteca Virtual em Saúde (BVS). 
Na base de dados PubMed, foi realizada a pesquisa por artigos no dia 20 de março de 2020, através das seguintes palavras-chave: COVID-19 and psychological consequences, COVID-19 and psychological impact of quarantine. O critério adotado para a escolha destas palavras-chave foram termos gerais com relação ao tema do estudo, a patologia COVID-19 e as consequências psicológicas decorrentes da quarentena. A princípio, foram encontrados 8 artigos, sendo posteriormente aplicados filtros de linguagem da própria base de dados para incluir estudos somente nos idiomas: português, inglês e espanhol. Nesta etapa, foram eliminados 3 artigos que não correspondiam a estes critérios de filtragem, restando 5 artigos. Como critérios de inclusão, utilizou-se estudos em humanos, transversais, caso-controle e coorte. Em relação aos critérios de exclusão foram: artigos de revisão de literatura, artigos duplicados e estudos não realizados em humanos. Neste sentido, foram eliminados 2 artigos duplicados, restando 3 artigos para a análise.

Em relação à plataforma de dados da BVS, foi realizada a pesquisa por artigos no dia 21 de março de 2020, por meio das seguintes palavras-chave: COVID-19 and psychological consequences, COVID-19 and psychological impact of quarantine. Os critérios adotados para a escolha das palavras-chave foram os mesmos utilizados na PubMed. Inicialmente, foram identificados 6 artigos e, logo em seguida, aplicou-se filtragem de linguagem da própria plataforma: português, inglês e espanhol, restando 3 artigos. Como critérios de inclusão, considerou-se estudos em humanos, transversais, caso-controle e coorte. Posteriormente, utilizou-se como critérios de exclusão: artigos de revisão de literatura, artigos duplicados e estudos não realizados em humanos. Nesse sentido, foram excluídos 1 estudo duplicado e 1 artigo de revisão de literatura. No final do processo de seleção, restou-se 1 artigo para a análise.

Nessa perspectiva, o corpus para a elaboração desta pesquisa, ao final, foi constituído por 4 artigos, sendo 3 da PubMed e 1 da BVS (Figura 1), cada artigo foi lido e analisado integralmente por dois revisores independentes a luz da saúde mental e o impacto da quarentena gerado pela pandemia da COVID-19, com o intuito de possibilitar a aplicabilidade desta revisão.

Figura 1. Representação esquemática da presente revisão integrativa

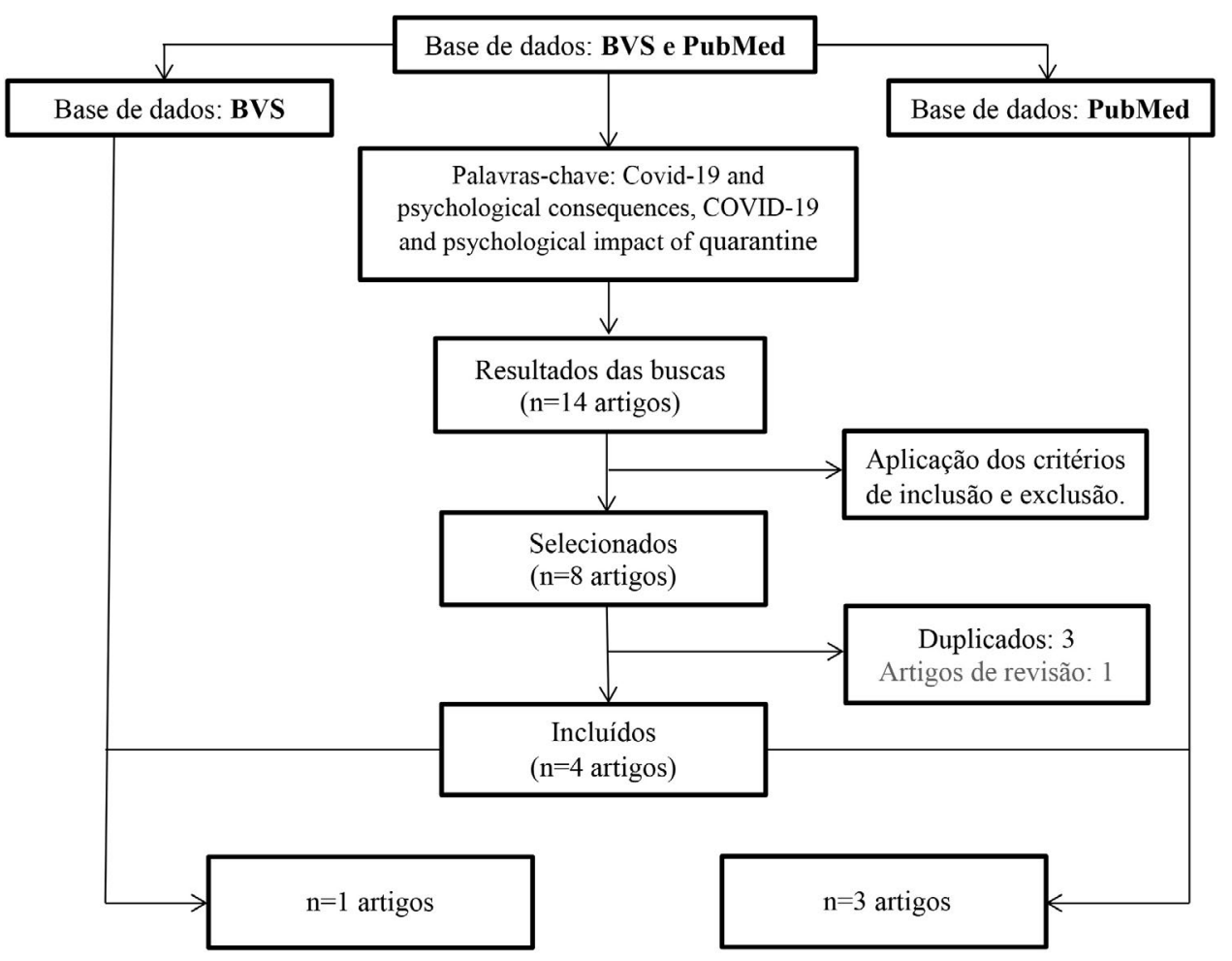


As informações obtidas nos artigos foram categorizadas, o conteúdo foi organizado e sumarizado em fichas de leitura individuais. As informações para cada estudo, no banco de dados, abrangeram o título do artigo, o autor, o ano de publicação, o método de análise e os resultados. Em seguida, realizou-se a análise crítica dos estudos incluídos na revisão integrativa. Os resultados foram analisados e interpretados criteriosamente, sendo agrupados em semelhanças e diferenças. Após a seleção dos artigos, com a constatação de diversos enfoques produtivos, selecionaram-se os principais pontos dos autores e ocorreu a conversão dos achados em uma forma visual de tabela exposto por meio da discussão textual e a síntese dos conteúdos da pesquisa.

Tabela 1. Informações técnicas dos artigos selecionados

\begin{tabular}{|c|c|c|c|}
\hline Título & Autores & Periódico & Local de publicação \\
\hline $\begin{array}{l}\text { A nationwide survey of psychological } \\
\text { distress among Chinese people in } \\
\text { the COVID-19 epidemic: implications } \\
\text { and policy recommendations }\end{array}$ & Qiu et al. & General Psychiatry & China \\
\hline $\begin{array}{l}\text { Progression of Mental Health } \\
\text { Services during the COVID-19 } \\
\text { Outbreak in China }\end{array}$ & Li et al. & $\begin{array}{l}\text { International Journal of } \\
\text { Biological Sciences }\end{array}$ & China \\
\hline $\begin{array}{l}\text { Factors Associated With Mental } \\
\text { Health Outcomes Among Health } \\
\text { Care Workers Exposed to } \\
\text { Coronavirus Disease } 2019\end{array}$ & Lai et al. & JAMA Network Open & China \\
\hline $\begin{array}{c}\text { The Impact of COVID-19 Epidemic } \\
\text { Declaration on Psychological } \\
\text { Consequences: A Study on Active } \\
\text { Weibo Users }\end{array}$ & Li et al. & $\begin{array}{l}\text { International Journal of } \\
\text { Environmental } \\
\text { Research and Public } \\
\text { Health }\end{array}$ & China \\
\hline
\end{tabular}

\section{Resultados e discussão}

A pandemia do coronavírus tem acarretado impacto amplo, substancial e duradouro ao psicológico da população, gerando ansiedade, medo, estresse, transtorno de pânico, depressão, solidão e angústia (Qiu et al., 2020; Li et al., 2020; Lai et al., 2020; Li et al., 2020). O estudo conduzido por Qiu et al. (2020), realizado com uma amostra de 52.730 pessoas, sob o efeito do isolamento social, identificaram que, aproximadamente, $35 \%$ dos pesquisados apresentaram transtornos psicológicos como transtorno do pânico, ansiedade e depressão. Dentre os estudos analisados, a incidência de patologias mentais variou, aproximadamente, entre 35 e $71 \%$ na população em geral (Tabela 2). 
Tabela 2. Apresentação dos métodos e resultados dos artigos selecionados

\begin{tabular}{|c|c|c|}
\hline Autores & Abordagem metodológica & Resultados \\
\hline Qiu et al. & $\begin{array}{l}\text { Realizou-se um questionário de autorrelato } \\
\text { denominado de COVID-19 Peritraumatic } \\
\text { Distress Index (CPDI) disponibilizado on-line } \\
\text { para a população em geral, a fim de analisar o } \\
\text { sofrimento psicológico gerado pela pandemia } \\
\text { do coronavírus. }\end{array}$ & $\begin{array}{l}\text { Dentre as } 52.730 \text { respostas obtidas, aproximadamente } 35 \% \text { da } \\
\text { amostra apresentaram transtornos psicológicos como } \\
\text { transtorno do pânico, ansiedade e depressão ocasionadas pelo } \\
\text { isolamento social. }\end{array}$ \\
\hline Li et al. & $\begin{array}{l}\text { Investigou-se as diretrizes e instruções para os } \\
\text { serviços de saúde mental durante o surto de } \\
\text { COVID-19 na China entre } 26 \text { de janeiro e } 20 \text { de } \\
\text { fevereiro de } 2020 \text {. }\end{array}$ & $\begin{array}{l}\text { Verificou-se a implantação de diretrizes no campo de saúde } \\
\text { mental como Manual Nacional de Saúde Mental; Plano de } \\
\text { Intervenção em Serviços de Saúde Psicossomática para } \\
\text { Prevenção e Controle do coronavírus e diretrizes para lidar } \\
\text { com a referida patologia, desenvolvido para populações } \\
\text { específicas (idosos, mulheres grávidas, profissionais de saúde etc. }\end{array}$ \\
\hline Lai et al. & $\begin{array}{l}\text { Estudo transversal pautado na avaliação dos } \\
\text { impactos psicológicos gerados pela pandemia } \\
\text { do coronavírus nos profissionais de saúde. A } \\
\text { amostra foi composta de } 1257 \text { profissionais de } \\
\text { saúde. Aplicou-se o Questionário de Saúde do } \\
\text { Paciente de } 9 \text { itens, Escala de } 7 \text { itens do } \\
\text { Transtorno de Ansiedade Generalizada; Índice } \\
\text { de Gravidade de Insônia de } 7 \text { itens e o impacto } \\
\text { de } 22 \text { itens da escala de eventos. }\end{array}$ & $\begin{array}{l}\text { A pesquisa apontou uma proporção considerável de } \\
\text { profissionais de saúde, com sintomas de depressão, } \\
\text { ansiedade, insônia e angústia, especialmente mulheres, } \\
\text { enfermeiras e profissionais de saúde de linha direta envolvidos } \\
\text { no diagnóstico, tratamento ou prestação de cuidados de } \\
\text { enfermagem a pacientes com suspeita ou confirmação. Uma } \\
\text { proporção considerável de participantes relatou sintomas de } \\
\text { depressão ( } 634 \text { [50,4\%]), ansiedade ( } 560 \text { [44,6\%]), insônia (427 } \\
\text { [34,0\%]) e angústia (899 [71,5\%]). }\end{array}$ \\
\hline Li et al. & $\begin{array}{l}\text { Compara-se a diferença antes e depois, de } 20 \\
\text { de janeiro, nas categorias linguísticas e no } \\
\text { perfil psicológico de postagens do Weibo. } \\
\text { Realizou-se a análise da amostra de } 17.865 \\
\text { usuários ativos, através da abordagem do } \\
\text { Reconhecimento Ecológico Online. }\end{array}$ & $\begin{array}{l}\text { Os resultados apontam que emoções negativas (ansiedade, } \\
\text { depressão e indignação) e a sensibilidade aos riscos sociais } \\
\text { aumentaram, enquanto os escores de emoções positivas } \\
\text { (felicidade e satisfação com a vida) diminuíram. Além disso, as } \\
\text { pessoas estavam mais preocupadas com sua saúde e família, } \\
\text { em comparação com lazer e amigos. }\end{array}$ \\
\hline
\end{tabular}

Por se tratar de grupos de risco com alta frequência de mortalidade, os idosos e pessoas com doenças crônicas apresentam maior probabilidade de desenvolver quadros psicopatológicos (Qiu et al., 2020). Todavia, vê-se que, na pesquisa de Sijia Li et al. (2020), os impactos da declaração da pandemia do COVID-19 em relação às consequências psicológicas de usuários ativos do Weibo, dos 17.865 usuários ativos, a mediana de idade foi de 33 anos e os resultados apontaram o aumento da incidência de emoções negativas (ansiedade, depressão e indignação) e a sensibilidade aos riscos sociais. Nesse sentido, notam-se lacunas relacionadas ao ciclo vital às condições psicopatológicas, sendo, portanto, imprescindível a compreensão de forma ampla acerca da problemática em questão. Assim, fica evidente a necessidade de política pública em saúde mental para amenizar os efeitos dessa pandemia.

Os profissionais de saúde por ocupar espaço na linha de frente do controle ao COVID-19 são, também, susceptíveis a serem acometidos por sintomatologias mentais. Nessa perspectiva, a pesquisa realizada por Lai et al. (2020) aos profissionais de saúde, em hospitais equipados com clínicas ou enfermarias para pacientes com COVID-19, em Wuhan e em outras regiões da China, notificou de 1257 pessoas que completaram o estudo, relataram sintomas de depressão (634 [50,4\%]), ansiedade (560 [44,6\%]), insônia (427 [34,0\%]) e angústia (899 [71,5\%]). Os profissionais de saúde de primeira linha envolvidos no diagnóstico, tratamento e atendimento diretos de pacientes com COVID-19 foram associados a um maior risco de desenvolvimento de resultados desfavoráveis à saúde mental. Isso sugere uma atenção diferenciada aos profissionais da linha de frente e a necessidade de apoio ou intervenções psicológicas. 
No que diz respeito às diferenciações de manifestações sintomatológicas entre os sexos feminino e masculino, verificou-se a presença de sintomas mentais, particularmente, sensações de angústias decorrentes da pandemia do novo coronavírus, em ambos os sexos com graus de afetação diferenciados. Averiguou-se que as mulheres tendem a apresentar maior vulnerabilidade quando expostas a situações de angústia como o cenário de instabilidade social desencadeado pela COVID-19, em relação à comparação com os homens, respectivamente com média (desvio-padrão) igual a 24,87(15,03) e 21,41 (15,97), $p<0,001$ (Qiu et al., 2020). É importante pontuar em paralelo, também, que os profissionais de saúde de primeira linha, do sexo feminino, relataram graus mais severos de sintomas de saúde mental (Lai et al., 2020). Isso evidencia que as mulheres, independente da atividade laboral e, também, as profissionais de saúde do sexo feminino, têm alto impacto emocional negativo frente ao coronavírus.

Observou-se, também, que pessoas com nível alto de escolaridade apresentaram maior nível de angústia nesse cenário de pandemia, variável que pode ser relacionada à função de autoconsciência mais aguçada sobre a saúde presente neste perfil de público. Foi averiguado que os trabalhadores migrantes tendem apresentar alta prevalência de angústia entre todas as ocupações. Identificaram-se escores altos de sofrimento mental em pessoas com idades entre 18 e 30 anos, eventualmente ligado ao acesso fácil às informações provenientes dos meios midiáticos que pode desencadear quadros de estresse, com média (desvio-padrão) igual a $21,41(15,97), p<0,001$. O grupo com menor risco de desenvolver patologias mentais são jovens com menos de 18 anos, possivelmente associado a baixa letalidade da doença nessa faixa etária e exposição restringida da pandemia devido à quarentena doméstica (Qiu et al., 2020).

Para conter o avanço da COVID-19, as autoridades públicas têm adotado medidas restritivas de isolamento social. Nesse ínterim, a dinâmica de organização e funcionamento da sociedade vem passando por profundas alterações e, concomitantemente, delineando mudanças nas rotinas diárias da população (Li et al., 2020). A instalação de medidas de isolamento social como restrição da circulação urbana, fechamentos de locais de entretenimento e lazer, proibição de aglomerações de pessoas, recomendações constantes para permanecer recluso em casa corroboram na criação do cenário de medo, ansiedade e solidão, gerando tensão psicológica. Assim, reduzida ou tolhida a capacidade de interação social, as pessoas são penetradas por pensamentos negativos de desamparo, permeando em suas vivências interiores sentimentos de solidão e tristeza (Qiu et al., 2020). Estas percepções psicológicas são comuns em situações de quarentena, evidenciando os danos mentais determinados pelo isolamento e restrição de contato humano.

Considerando o homem como ser naturalmente social, as interações humanas exercem papel importante como fator de suporte psicossocial (Carvalho \& Matos, 2016). Partindo dessa perspectiva, a ausência de contato humano pode se tornar um fator de risco para delineamento de sofrimento existencial e social. Desta forma, a dificuldade de estabelecimento do suporte psicossocial de familiares e amigos gerada pela barreira física da quarentena fomenta implicações na redução da capacidade de resposta diante das intempéries presentes nesse cenário de pandemia (Qiu et al., 2020). A ausência de rede psicossocial em momentos de crise seja relacionada à saúde, economia ou política, contribuem para o delineamento e agravamento de quadros clínicos de sofrimento mental.

Nessa perspectiva, as interações sociais são importantes para a constituição humana, visto que as relações entre as pessoas são fontes de aprendizados necessários para o desenvolvimento do homem em sua integralidade biopsicossocial (Carvalho \& Matos, 2016). No entanto, as medidas de isolamento social ocasionaram a ruptura desse ciclo de interações humanas, fomentando condições limitantes para a comunicação entre as pessoas. As restrições de interações sociais desencadearam diferentes formas de subjetivação e afetação negativa na dinâmica existencial. A impossibilidade de interação face a face entre as pessoas culminou na configuração de processos intrincados de sofrimento psicológico em diferentes perfis, idades e classes sociais, indistintamente. $O$ esfacelamento dos elos sociais que conecta fisicamente as pessoas conduziu a formação de sensações de desamparo, reverberando na construção de espaço fecundo para a solidão, ansiedade e tristeza (Qiu et al., 2020). 
Diante desse cenário, caracterizado pela instabilidade e insegurança, as pessoas vivenciam surtos psicológicos, desencadeados pelo medo, solidão e pânico coletivo. Essas mudanças no cotidiano da população decorrente da quarentena ocasionam dificuldades adaptativas acompanhadas por desorganização social e mental (Li et al., 2020). Nesse contexto, as pessoas são atravessadas diariamente por sensações de insegurança e o medo iminente de serem infectadas pelo vírus e, por vezes, convivem com a falta de previsão para o retorno à vida normal, delineando condições favoráveis para o adoecimento mental.

Para além das medidas restritivas de isolamento social, temos como fatores que ampliam a margem de sofrimento mental no público em geral: o pânico coletivo gerado pela divulgação diária de notícias em meios midiáticos acerca do coronavírus, número crescente de casos diagnosticados com a patologia, o aumento da taxa de letalidade, perdas de entes queridos e impossibilidade de visitar amigos e familiares (Qiu et al., 2020). Neste sentido, negligencia-se a saúde mental mundialmente.

No tocante a quarentena, trata-se de contexto propício para desencadeamento e agravamento de transtornos mentais (Qiu et al., 2020). Nesse sentido, faz-se necessário destacar as possíveis variáveis que são estressores durante a quarentena: duração da quarentena, as mais longas são associadas especificamente a problemas de saúde mental; sintomas de estresse pós-traumático; comportamentos de esquiva e raiva; medos de infecção; temores acerca da própria saúde ou medo de infectar, frustração e tédio; o confinamento, a perda da rotina habitual e o contato social e físico reduzido com os outros; suprimentos inadequados; e, ausência de informações concisas referentes às autoridades de saúde públicas. Ressaltam-se, também, os estressores pós-quarentena que estão relacionadas às finanças e ao estigma de pessoas que foram colocadas em quarentena (Brooks et al., 2020). É preciso compreender que a saúde mental e econômica são parceiras harmônicas e inseparáveis.

Nessa perspectiva, o isolamento social acompanhado do medo e insegurança diante da possibilidade iminente de transmissão do vírus tem fomentado implicações para a saúde mental da população em geral, favorecendo na criação de condições psicopatológicas que interfere na forma como o sujeito se sente, pensa e age e nas suas relações consigo mesmo, com os outros e o mundo. Por se tratar de uma patologia viral que não tem uma terapia farmacológica eficiente e acompanhada pela incerteza da cura, o coronavírus gera tensões psicológicas na vida das pessoas que convivem diariamente com o medo iminente de serem infectadas, reverberando a configuração de um pré-adoecimento, isto é, a doença provoca sofrimento antes mesmo de instalar-se no organismo (Qiu et al., 2020).

O distanciamento social provocado pela pandemia da COVID-19 mostrou as lacunas da fragilidade humana, com repercussões mais severas em pessoas que apresentam relações socioemocionais esfaceladas e frágeis, dificuldades econômicas e instabilidade no vínculo empregatício e condições inapropriadas de habitação que impossibilitam manter a quarentena doméstica (MS, 2020). Este último aspecto reitera a desigualdade social como mecanismo limitante para acesso aos recursos adequados de proteção à saúde de pessoas em condições econômicas desfavoráveis nesse contexto de pandemia ( $\mathrm{Li}$ et al., 2020). Em geral, esses fatores dificultam a adesão das pessoas às medidas de isolamento social (MS, 2020).

As medidas de quarentena fomentam, também, implicações econômicas, que porventura geram ansiedade e angústia. Diante desse cenário, pessoas com vínculo empregatício sentem medo de serem demitidas pelas empresas, enquanto os autônomos se encontram na aflição diária de não conseguirem recursos financeiros para pagamento de suas despesas ou de sua família. Além da possibilidade de perda financeira, a população pode vivenciar percepções psicológicas como tédio, decepção, e irritabilidade, resultado das medidas de isolamento (Li et al., 2020). A saúde, nominalmente, conceitua-se como o mínimo atendimento aos direitos sociais, segundo o Grupo de Estudo e Pesquisa em saúde/doença e direitos sociais da Universidade Estadual da Paraíba, Brasil. (grifo nosso)

No que se refere às pessoas diagnosticadas com COVID-19, podem apresentar sintomatologias psicopatológicas como negação, solidão, medo, depressão, insônia, desespero e ansiedade, em função das consequências graves que essa patologia apresenta no organismo, podendo até reduzir a adesão ao tratamento (Li et al., 2020). Qiu et al. (2020) esclarecem 
que esses pacientes podem assumir comportamentos de risco e chegar a cometerem suicídio ou agressão. As pessoas com suspeita de COVID-19 podem desenvolver sintomas de ansiedade e transtornos obsessivo-compulsivos com tendência frenética e repetitiva de verificar temperatura corporal e limpeza das mãos, influenciado pelo estado de incerteza da sua saúde ( $\mathrm{Li}$ et al., 2020). Vê-se, de forma clara, a necessidade de cuidado em saúde mental frente à COVID-19.

Partindo dessa perspectiva, a pandemia do coronavírus tem fomentado um cenário propício para desencadeamento e potencialização de psicopatologias, ampliando a demanda por serviços psicológicos como psicoterapias e escutas psicológicas (Qiu et al., 2020). Diante dessa nova realidade social, os serviços de saúde mental que eram até então presenciais houve a necessidade de serem revisados e propostas novas modalidades de atendimento nessa conjuntura de pandemia. Como forma de atender às demandas psicológicas, organizações de saúde e profissionais em saúde mental desenvolveram serviços assistência mental on-line como aconselhamento mental via plataformas eletrônicas e exposição de vídeos educacionais em saúde pública para profissionais de saúde mental e o público em geral (Li et al., 2020). No entanto, averígua-se a presença de lacunas nessas medidas de assistência on-line, visto que nem toda população se beneficia dessa modalidade de serviço, devido à falta de acesso aos recursos informativos como internet, computadores ou celulares, principalmente pessoas de baixa renda ou sujeitos com conhecimentos limitados dessas tecnologias.

Segundo Li et al. (2020), as intervenções no âmbito da saúde mental deve se pautar em três princípios: compreensão do estado psicológico da pessoa, identificação dos grupos mais vulneráveis ao desenvolvimento de transtornos mentais e a construção de intervenções psicológicas adequadas às demandas de cada perfil populacional. Neste sentido, ações em saúde mental são desenvolvidas a partir das demandas potencialmente identificadas, considerando a singularidade do sujeito e o contexto à qual está inserido como eixos centrais para elaborar o plano de intervenção. Considerando o sujeito em sua condição biológica, psicológica, social e espiritual, a prática em saúde mental frente à emergência de pandemia, como a COVID-19, articula-se na confecção de estratégias que atravessam todas essas dimensões e compreendem a pessoa em sua integralidade, fundamentando numa atenção holística baseada na escuta empática, acolhedora, compreensiva e, sobretudo, promotora de bem-estar e qualidade de vida (Cardoso \& Galera, 2011). O correto a afirmar na atualidade, possivelmente, o mundo não estava preparado mentalmente para pandemia.

No cenário chinês, Qiu et al. (2020) apontam três fatores presentes na pandemia do COVID-19 que podem ter contribuído para causar pânico coletivo: a confirmação do primeiro caso de transmissão de COVID-19 por contato entre humanos pelas autoridades de saúde, a instalação da quarentena estrita de Wuhan em 22 de janeiro e anúncio da Organização Mundial de Saúde da emergência de saúde pública de preocupação internacional em 31 de janeiro (WHO, 2020). Contextualizando essas informações para o cenário brasileiro, podemos destacar o dia em que foi anunciado pelo Ministério da Saúde o primeiro caso notificado de COVID-19 em São Paulo (MS, 2020) e junto com a notícia emergiu um clima de pânico e ansiedade pela população. Logo após, com o crescimento do número de pessoas infectadas, autoridades públicas adotaram medidas de isolamento social, gerando um caos social e ansiedade generalizada na sociedade.

Para minimizar os impactos psicológicos do coronavírus, Brooks et al. (2020) mencionam a importância de os governos manterem informados a população acerca de todas as medidas adotadas, explicando o porquê de elas serem realizadas, assim como desenvolver atividades prazerosas para serem realizadas durante a quarentena e, sobretudo, garantir suprimentos básico e atenção especial aos profissionais de saúde. Registra-se no Brasil, o preparo emocional destes profissionais para o controle da pandemia mesmo diante do quadro de baixo reconhecimento financeiro e social. Apesar das dificuldades presentes nesse contexto de pandemia (insuficiência de equipamentos de proteção, riscos de contágio pela patologia, carga excessiva de trabalho, entre outros), esses profissionais têm assumido uma posição ativa no tratamento de pacientes com diagnósticos positivos do coronavírus, prestando assistência à população mesmo sendo mal pagos e invisíveis para a sociedade.

A estabilização do quadro de transmissão do coronavírus no território chinês, segundo Qiu et al. (2020), está relacionada a adoção de medidas eficazes de prevenção e controle estabelecidas pelo governo chinês como os quartéis de assistência médica, construção de diretrizes e manuais destinados à população como recurso informativo, fortalecimento da proteção 
individual, isolamento médico, restrição a circulação urbana, proibição de aglomerações de pessoas. O Brasil tem adotado algumas dessas medidas como forma de conter o avanço do vírus no cenário nacional (MS, 2020; WHO, 2020; Casa Civil [CC], 2020). Por enquanto, é muito cedo afirmar se essas medidas serão tão benéficas como na China. A curva de contágio da doença no Brasil tem crescido conforme o tempo passa, colocando o país num patamar preocupante no cenário internacional, à qual caminha em direção para tornar-se um novo epicentro dessa pandemia. (WHO, 2020; WHO, 2020; WHO, 2020). No entanto, é importante frisar que o número de brasileiros infectados pelo coronavírus pode ser muito maior do que é registrado nas estatísticas oficiais, sendo suscetível a existência de subnotificações da patologia no cenário nacional, podendo ser um fator limitante para o enfrentamento sistemático desse fenômeno patológico.

Considerando a rápida proliferação do COVID-19, autoridades públicas e organizações de saúde elaboraram diretrizes e instruções para nortear os serviços de saúde mental como forma de minimizar os impactos psicológicos gerados pela patologia no cenário social. O estudo de Li et al. (2020), na revisão acerca das diretrizes adotadas no cenário chinês no âmbito da saúde mental, destacou como medidas importantes: Manual Nacional de Saúde Mental com informações nosológicas sobre o coronavírus; Diretrizes para lidar com a referida patologia, desenvolvido para populações específicas (idosos, adultos, crianças e adolescentes, mulheres grávidas e profissionais de saúde); Plano de Intervenção em Serviços de Saúde Psicossomática para Prevenção e Controle do Coronavírus; Protocolo de diagnóstico e tratamento para o COVID-19, com ênfase nas medidas de isolamento das enfermeiras destinadas aos pacientes com a referida patologia e redução das consultas ambulatoriais; publicação do documento denominado de "Princípios para Crises Psicológicas de Emergência e Intervenção para a epidemia de pneumonia COVID-19" em 27 de janeiro de 2020; e Plano de Prevenção de doenças mentais estabelecido pela Comissão Nacional de Saúde da China (NHC). No que diz respeito às pessoas com diagnóstico de transtornos mentais, Li et al. (2020) apontaram iniciativas das autoridades de saúde para o tratamento comunitário para tais pacientes em suas residências. Essas diretrizes e instruções podem ser adotadas no contexto brasileiro como ações voltadas à promoção, prevenção e tratamento em saúde mental.
No Brasil, a adoção de medidas no campo de saúde mental ainda é incipiente, não se tem percebido ações efetivas por parte das autoridades públicas que agrega essa dimensão como um dos eixos centrais de políticas públicas assistenciais. Revisando as medidas adotadas pelo governo federal desde fevereiro até abril de 2020, notam-se lacunas no plano de intervenção voltado à promoção e prevenção de saúde mental. Observam-se ações mais direcionadas ao tratamento e medidas de contenção ao avanço do vírus (CC, 2020). Dentre tais ações, podem-se elencar a criação de Campanhas Publicitárias voltadas à prevenção do Coronavírus e o desenvolvimento do aplicativo (app) Coronavírus-Sus como forma de fornecer assistência e orientação à população (MS, 2020).

Por outro lado, observam-se algumas iniciativas de instâncias públicas no campo de saúde mental como a criação do Manual de Saúde Mental e Atenção Psicossocial na Pandemia COVID-19, resultado da parceria realizada entre Ministério da Saúde e Fundação Oswaldo Cruz, sugerindo recomendações para gestores na atuação em saúde mental (MS, 2020). A elaboração de manuais de instrução e diretrizes no campo de saúde mental serve para orientar e informar a população sobre modos de lidar com esse cenário de pandemia, elencando medidas para a preservação da integridade física e mental da população. No âmbito da saúde mental, houve ainda as recomendações do Ministério da Saúde para atendimento psicológico on-line.

Apesar dessas iniciativas, vê-se a necessidade de ampliação de políticas públicas e projetos no campo de assistência mental. Por conseguinte, reitera-se a limitação de ações por parte de autoridades públicas no âmbito da saúde mental e as implicações negativas para a assistência integral ao público em geral, que demanda cuidado para além da dimensão biológica, necessitando de atenção psicossocial. Neste sentido, inclui-se a manutenção de trabalho, emprego e renda dos brasileiros (Pachú, 2015), bem como fortalecimento de redes socioafetivas e serviços assistenciais de suporte psicológico como psicoterapias e escutas psicológicas.

O presente estudo registrou correlação significativa entre a pandemia do coronavírus e os impactos gerados a saúde mental da população. Averiguou-se a existência de prejuízos psicológicos influenciados pelo cenário pandêmico da COVID-19, criando espaço favorável para desencadeamento e agravamento de patologias mentais como depressão, ansiedade, insônia, angústia, estresse e transtorno de pânico (Qiu et al., 
2020; Li et al., 2020; Lai et al., 2020; Li et al., 2020). O aglomerado de informações emitidas nos meios midiáticos acerca da referida patologia, a rápida disseminação do vírus, a ausência de medidas eficientes de assistência psicossocial desenvolvida pelas instâncias públicas se apresenta como fatores que ampliam os efeitos nocivos da patologia na saúde mental da sociedade. Ademais, sugere-se a construção de estratégias de promoção da saúde mental como forma de minimizar os impactos psicológicos gerados pela quarentena decorrente da pandemia da COVID-19.

\section{Conclusões}

Para compreensão dos impactos gerados pela COVID-19 na coletividade se faz necessário considerar os fatores de natureza biológica, psicológica e social que compõe a dinâmica organizacional de ordem nosológica, taxonômica e semântica desse fenômeno patológico que perpassa nesse processo de adoecimento. A partir disso, verificou-se a possibilidade de transitar por diferentes cenários e contextos que delineia a conjuração de linhas que interferem nas instâncias inter, intra e transubjetivo.

Considerando o aspecto psicológico como dimensão que circunda e interfere nas demais, mostra-se o seu papel fundamental para inferência de processos intrincados de sofrimento existencial e social, sendo uma das dimensões mais afetadas pelas repercussões da COVID-19. O eco que entoa em cada novo caso notificado de COVID-19 alerta as pessoas para sua condição de vulnerabilidade e risco iminente de morte, gerando desespero e ansiedade.

Na presente pesquisa, ficou evidente a maior frequência de relatos de reações e sintomas possivelmente associados a transtornos mentais, tais como queixas de ansiedade, depressão, insônia, estresse, pânico, solidão, angústia, obsessão e compulsão, entre outros. Os estudos analisados sugerem que a pandemia do coronavírus fomenta implicações para além da dimensão meramente biológica, podendo também ser uma fonte de sofrimento mental.

As medidas de isolamento de social adotadas para conter o avanço da COVID-19 ampliaram a margem para o sofrimento mental, haja vista que modificaram bruscamente a rotina diária da população em diferentes esferas da vida como trabalho, educação, interação comunitária, lazer, entre outros, perfazendo o delineamento de dificuldades adaptativas à nova realidade social. Nesse contexto, as pessoas foram submetidas a uma série de elementos estressores como sensações de insegurança, o medo iminente de serem infectadas pelo vírus e infectar seus familiares, dificuldades para a obtenção de apoio socioemocional, falta de suprimentos adequados para a proteção física contra a COVID-19, e a convivência diária com a incerteza e sem a previsão para o regresso à vida normal, conduzindo para o desenvolvimento de sofrimento existencial e social.

Grupos de risco como idosos e pessoas com doenças crônicas, assim como profissionais de saúde, trabalhadores migrantes, mulheres e pessoas com alto nível de instrução educacional, apresentaram maior probabilidade para desenvolvimento de quadros clínicos de sofrimento mental. Enquanto, jovens com menos de 18 anos têm a menor prevalência de sintomas mentais. Daí a inquestionável e necessária adoção de medidas de promoção, prevenção e tratamento em saúde mental.

Desse modo, são inúmeros os desafios relacionados à promoção da saúde mental no contexto de pandemia do coronavírus. Destacando-se as informações referentes ao período de quarentena, sofrimento psíquico diante do isolamento social e possibilidade de infecção, o desenvolvimento de quadros psicopatológicos, o impacto psicológico pós-quarentena, o cuidado com profissionais da área de saúde e a ausência de suprimentos básicos a coletividade. Ademais, salienta-se que, a política de saúde mental deverá abraçar outras classes de trabalhadores que se mantêm em plena atividade durante a pandemia no Brasil: motoristas, policiais, colaboradores de supermercados, postos de gasolina, farmácias e limpeza urbana.

A partir dos resultados supracitados, faz-se necessário ampliar as medidas interventivas frente ao aumento da incidência de transmissão do vírus na sociedade, exigindo dos profissionais de saúde e autoridades públicas a capacidade criativa e inovadora de cultivar ações de promoção da saúde diante deste universo de medo, angústia, ansiedade e solidão que aflige a vida da população. Interpõem-se a importância do trabaIho coletivo, partilhado, construído em bases edificantes que interligam diversos atores sociais na constituição dinâmica vinculada aos processos saúde/doença. Assim, fortalecendo as possibilidades em saúde mental apesar das intempéries e encalços presentes nesse cenário de insegurança e instabilidade social. 
Como recomendações para elaboração de estratégias interventivas no âmbito da saúde destacam-se: atenção especial das autoridades de saúde aos grupos vulneráveis como idosos e pessoas com doenças crônicas; adoção de políticas públicas assistenciais de promoção da saúde mental para o público em geral; melhoria dos serviços de assistência para atender as demandas provenientes da pandemia do coronavírus; ampliação de oferta de serviços psicológicos como psicoterapia e escuta psicológica; aplicação de estratégias de monitoramento epidemiológico; transparências das informações e medidas adotadas para o enfrentamento dessa pandemia. Por fim, e não menos importante, o planejamento estratégico de ações de assistência psicológica destinados aos profissionais de saúde que tem sob seu gerenciamento diversas vidas para cuidar e devem receber uma atenção integral do governo, à promoção do cuidado daqueles que cuidam.

Como limitação dessa pesquisa, mostrou-se o número reduzido de artigos encontrados nas bases de dados PubMed e Biblioteca Virtual em Saúde. No entanto, pelos resultados encontrados, fica demonstrado a necessidade da ampliação do número de pesquisas qualitativas a respeito dos diversos aspectos da COVID-19 e implementação de políticas públicas acerca de cuidados na saúde mental da população.

Ademais, verificou-se que as discussões acerca do coronavírus encontram-se de forma incipiente no cenário científico brasileiro, destacando-se, assim, a necessidade de elaboração de produções científicas posteriores para entender as implicações do COVID-19 na saúde mental. Seja de pacientes, profissionais de saúde e do público em geral, a fim de subsidiar a construção de políticas públicas assistenciais voltadas à promoção, prevenção e tratamento, no âmbito da saúde.

Há, ainda, pouco acervo na literatura científica que discute os impactos psicológicos gerados pela quarentena decorrente da pandemia do coronavírus, sendo, assim, necessário encorajar pesquisas que contemple essa área de estudo no cenário nacional. A presente pesquisa abre espaço para realização de novos estudos e, também, fomenta contribuições para a elaboração de estratégias de promoção da saúde mental em termo de políticas públicas, como forma de minimizar os impactos psicológicos gerados pela pandemia da COVID-19.

\section{Contribuições dos autores}

Lira AVAA contribuiu na produção escrita referente à introdução, metodologia, resultados e discussão e conclusões, corrigiu e revisou a versão final do texto. Pereira NAC elaborou as ilustrações e tabelas, colaborou na elaboração dos resultados e discussão. Ramos LAIA realizou a pesquisa bibliográfica e revisão final do texto. Pachú CO corrigiu e revisou a versão final do texto.

\section{Conflitos de interesses}

Nenhum conflito financeiro, legal ou político envolvendo terceiros (governo, empresas e fundações privadas, etc.) foi declarado para nenhum aspecto do trabalho submetido (incluindo, mas não se limitando a subvenções e financiamentos, participação em conselho consultivo, desenho de estudo, preparação de manuscrito, análise estatística, etc.).

\section{Referências}

Brooks, S. K., Webster, R. K., Smith, L. E., Woodland, L., Wessely, S., \& Greenberg, N. (2020). The psychological impact of quarantine and how to reduce it: rapid review of the evidence [O impacto psicológico da quarentena e como reduzi-lo: revisão rápida das evidências]. The Lancet, 395(10227), 912-920. https://doi.org/10.1016/S0140$\underline{6736(20) 30460-8}$

Cardoso, L, \& Galera, S. A. F. (2011). O cuidado em saúde mental na atualidade. Revista da Escola de Enfermagem da USP, 45(3), 687-91. https://doi.org/10.1590/50080$\underline{62342011000300020}$

Carvalho, M. A. D., \& Matos, M. M. G. (2016). Psychosocial Interventions in Crisis, Emergency and Catastrophe [Intervenções psicossociais em crises, emergências e catástrofes]. Revista Brasileira de Terapias Cognitivas, 12(2), 116-125. http://dx.doi.org/10.5935/1808-5687.20160018

Casa Civil. (2020). Presidência da República. Medidas adotadas pelo Governo Federal no combate ao coronavírus - 2 de abril. Gov.br. https://www.gov.br/casacivil/pt-br/assuntos/ noticias/2020/abril/medidas-adotadas-pelo-governofederal-no-combate-ao-coronavirus-2-de-abril

Huang, C., Wang, Y., Li, X., Ren, L., Zhao, J., Hu, Y., Zhang, L., Fan, G., Xu, J., Gu, X., Cheng, Z., Yu, T., Xia, J., Wei, Y., Wu, W., Xie, X., Yin, W., Li, H., Liu, M.,... Cao B. (2020). Características clínicas de pacientes infectados com o novo coronavírus de 2019 em Wuhan, China [Características clínicas de pacientes infectados com o novo coronavírus de 2019 em Wuhan, China]. Lancet, 15;395, 497-506. https://doi. org/10.1016/S0140-6736(20)30183-5 
Lai, J., Ma, S., Wang, Y., Cai Z., Hu J., Wei N., Wu, J., Du, Hui., Chen, T., Li, R., Tan , H., Kang, L., Yao, L., Huang, M., Wang, H., Wang, G., Liu, Z., \& Hu S. (2020). Factors Associated With Mental Health Outcomes Among Health Care Workers Exposed to Coronavirus Disease 2019 [Fatores associados aos resultados de saúde mental entre profissionais de saúde expostos à doença do coronavírus em 2019]. Jama Network Open, 3(3), 1-12. https://doi.org/10.1001/ jamanetworkopen.2020.3976

Li, W., Yang, Y., Liu., Z. H., Zhao, Y. J., Zhang, Q., Zhang, L., Cheung, T., \& Xiang, Y. T. (2020). Progression of Mental Health Services during the COVID-19 Outbreak in China [Progressão dos serviços de saúde mental durante o surto COVID-19 na China]. International Journal of Biological Sciences, 16(10), 1732-1738. https://doi.org/10.7150/ ijbs. 45120

Li, S., Wang, Y., Xue, J., Zhao, N., \& Zhu, T. (2020). The Impact of COVID-19 Epidemic Declaration on Psychological Consequences: A Study on Active Weibo Users [O impacto da declaração sobre epidemias de COVID-19 sobre consequências psicológicas: um estudo sobre usuários ativos do Weibo]. International Journal of Environmental Research and Public Health, 17(2032), 1-9. https://doi. org/10.3390/ijerph17062032

Marques, F. (2020). A quarentena na COVID-19: Orientações e estratégias de cuidado. Fiocruz. https:// www.fiocruzbrasilia.fiocruz.br/a-quarentenana-covid-19-orientacoes-e-estrategias-decuidado/\#: :text=A\%20nova\%20cartilha\%20aborda\%20 orienta\%C3\%A7\%C3\%B5es,mental\%20daqueles\%20que\%20as\%20experienciam

Ministério da Saúde. (2020). Brasil confirma primeiro caso da doença. Gov.br. https://www.gov.br/saude/pt-br/ assuntos/noticias/brasil-confirma-primeiro-caso-de-novocoronavirus

Ministério da Saúde. (2020). Doença pelo Coronavírus 2019: Atualização das Definições de Casos. Boletim Epidemiológico, 2020. https://portalarquivos2.saude. gov.br/images/pdf/2020/marco/04/2020-03-02-BoletimEpidemiol--gico-04-corrigido.pdf

Ministério da Saúde. (2020). Fiocruz. Recomendações para gestores. Fiocruz Brasília. https://www.fiocruzbrasilia. fiocruz.br/wp-content/uploads/2020/04/SaúdeMental-e-Atenção-Psicossocial-na-Pandemia-Covid-19recomendações-para-gestores.pdf

Pachu, C. O. (2015). Direitos sociais: o Artigo 6 da Constituição Federal e sua efetividade. EDUEPB. http://editora.ifpb.edu. br/index.php/uepb/catalog/view/140/111/370-1
Qiu, J., Shen, B., Zhao, M., Wang Z., Xie B., \& Xu, Y. (2020). A nationwide survey of psychological distress among Chinese people in the COVID-19 epidemic: implications and policy recommendations [Uma pesquisa nacional de sofrimento psicológico entre os chineses na epidemia de COVID-19: implicações e recomendações de políticas]. General Psychiatry, 33(2), 1-3. https://doi.org/10.1136/ gpsych-2020-100213

Souza, M. T., Silva, M. D, \& Carvalho, R. (2010). Revisão integrativa: o que é e como fazer. Einstein, 8(1), 102-106. http://dx.doi. org/10.1590/s1679-45082010rw1134

Wilder-Smith, A., \& Freedman, D. O. (2020). Isolation, quarantine, social distancing and community containment: pivotal role for old-style public measures in the novel coronavirus (2019-nCoV) outbreak [Isolamento, quarentena, distanciamento social e contenção da comunidade: papel fundamental para medidas públicas de estilo antigo no novo surto de coronavírus (2019-nCoV)]. J Travel Med, 27(2), 1-4. https://doi.org/10.1093/jtm/taaa020

World Health Organization. (2020). Coronavirus disease 2019 (COVID-19) Situation Report - 43 [Relatório da situação de doença coronavírus 2019 (COVID-19) - 43]. https://www. who.int/docs/default-source/coronaviruse/situationreports/20200303-sitrep-43-covid-19.pdf

World Health Organization. (2020). Coronavirus disease 2019 (COVID-19): Situation Report - 73. latest survey of recorded cases of covid-19 by the world health organization [Doença por coronavírus 2019 (COVID-19): Relatório da situação - 73. última pesquisa de casos registrados de covid-19 pela organização mundial de saúde]. https://www. who.int/docs/default-source/coronaviruse/situationreports/20200402-sitrep-73-covid-19.pdf

World Health Organization. (2020). Report of the WHO-China Joint Mission on Coronavirus Disease 2019 (COVID-19). who.int. https://www.who.int/docs/default-source/coronaviruse/ who-china-joint-mission-on-covid-19-final-report. pdf?sfursn=fce $87 f 4$ e_ 2

World Health Organization. (2020). Pneumonia of unknown cause - China. who.int. https://www.who.int/csr/don/05-january2020-pneumonia-of-unkown-cause-china/en/

World Health Organization. (2020). Rolling updates on coronavirus disease (COVID-19). who.int. https://www.who.int/ emergencies/diseases/novel-coronavirus-2019/events-asthey-happen

World Health Organization. (2020). WHO Timeline - COVID-19. who.int. https://www.who.int/news-room/detail/08-042020-who-timeline---covid-19

World Health Organization. (2020). Virtual press conference on COVID-19: 11 March 2020. who.int. https://www.who.int/ docs/default-source/coronaviruse/transcripts/who-audioemergencies-coronavirus-press-conference-full-and-final11 mar2020.pdf?sfvrsn=cb432bb3 2 